\title{
The categoricity problem and truth-value gaps
}

\section{IAN RuMfiTT}

In a recent paper, ${ }^{1} \mathrm{~T}$. J. Smiley has revived, and advanced a solution to, an important but neglected problem in logical theory - that of finding 'categorical' axiomatizations for logical calculi. Using rules of rejection, he solves the problem for the classical propositional calculus, and for predicate and modal systems based upon it. As I show, however, his method of axiomatization breaks down over calculi that permit truth-value gaps breaks down, indeed, even over the central cases of compact calculi that classify sentences simply as true, as false, or as neither. I produce a method that handles these cases, and illustrate it by giving a categorical axiomatization for a propositional fragment of Smiley's own (1960) free predicate calculus.

\section{The categoricity problem, and Smiley's solution to it}

In understanding what the categoricity problem is, and why it matters, it helps to consider a popular and plausible view of the meaning of the logical constants. On this view, most persuasively expounded by Hacking (1979), the introduction rules (and/or the elimination rules) which govern the logical constants in a sound and complete deduction system for a calculus ${ }^{2}$ are understood as giving the meaning of those constants (as they are used in that calculus). Those who adopt this position typically understand 'sound and complete' to mean 'sound and complete in respect of the consequence relation'. ${ }^{3}$ That is to say, they have in mind systems in which a formula $A$ is deducible from a set of formulae $X$ just when every admissible valuation of the calculus that verifies all the members of $X$ also verifies $A .{ }^{4}$ However,

1 Smiley 1996. All unqualified page references are to this article.

2 Following Smiley, I shall call such a system an axiomatization of the calculus. The term is somewhat unhappy: in many contexts, it is important to distinguish between axioms and rules. But it is widespread, and I know of no better.

3 Although Hacking's own position is subtly, but importantly, different; see fn. 8 below.

4 The admissibility of a valuation may of course vary from calculus to calculus. I say that a valuation verifies a formula $A$ when it evaluates $A$ as true, falsifies $A$ when it evaluates $A$ as false, fails to verify $A$ when it does not evaluate $A$ as true, etc. 
when understood in this way, the view is untenable. For consider (1) the standard two-valued propositional calculus alongside (2) the calculus that differs from (1) only inasmuch as it admits the valuation that verifies every formula of the relevant propositional language. As Smiley observes, 'logical consequence is the same relation in calculi (1) and (2), for it depends exclusively on the impossibility of the premisses being true and the conclusion false' - i.e. on the calculus's not admitting a valuation that verifies all the premisses while falsifying the conclusion - 'and the possibility or otherwise of their all being true is irrelevant' (p. 7). Accordingly, any deduction system that is sound and complete (in the present sense) with respect to calculus (1) will also be sound and complete with respect to calculus (2), so that the standard introduction and elimination rules for ' $\sim$ ' are compatible with an interpretation whereby ' $\sim$ ' behaves as in calculus (2). But in calculus (2) $P$ and $\sim P$ can both be true, i.e. are not so much as contraries. This surely shows that the standard rules fail to capture an important aspect of the intended meaning of ' $\sim$ '. Indeed, as Smiley notes, the problem here generalizes, for 'the device by which (1) and (2) are differentiated can be applied anywhere, so axiomatization of the usual sort can never exclude unintended interpretations of any calculus' (p. 8). We have, then, a general 'failure of categoricity at the sentential level' (ibid.).

Smiley's paper presents a solution that preserves the spirit of the popular and plausible view. He begins by introducing into the language a primitive sign of rejection, ' $"$ ', which is used in such a way that ' $P$ ' 'indicates dissent from the proposition expressed by $P^{\prime}$ (p. 2). The star may be attached to any formula of the original language, but as an indicator of force it cannot be iterated. With the language so enlarged, and where the members of $X$, and $A$, are now starred or unstarred formulae, ${ }^{5}$ we may define the Smiley consequence relation $\vDash$ of a calculus to be the relation for which $X \vDash A$ iff every admissible valuation of the calculus that verifies all the formulae that appear unstarred in $X$, and falsifies all the formulae that there appear starred, verifies the formula in $A$ (should it appear unstarred) or falsifies it (should it appear starred) (cf. p. 5). All the traditional logical notions are definable in terms of Smiley consequence. For example, formulae $P$ and $Q$ are contraries iff $P \vDash * Q$, sub-contraries iff $* P \vDash Q$, contradictories iff

5 Even though there is no perceptible difference between them, it is crucial to understanding Smiley's treatment that one keep in mind the categorial difference between a formula tout court and an unstarred (or asserted) formula. With some misgivings, but in order to ease comparison with his treatment, I shall follow him in not making this difference formally perspicuous, relying instead upon context, and explanatory glosses, to fix the intended meaning. Those who disapprove will have no trouble making the relevant difference in category perspicuous, for example by reviving the Fregean 'use of the turnstile to indicate assertion [so that] * $P$ and $\vdash P$ are immediately seen to be co-ordinate items' (p. 6). 
$P \vDash{ }^{*} Q$ and vice versa (p. 8). More generally, as he also shows (ibid.), the relation of Smiley consequence suffices to determine the entire sentential output of the calculus. That is to say, it determines the classification of every assignment of truth values (across all the formulae of the calculus simultaneously), as being either admissible or inadmissible (cf. p. 7). Now the sentential output of a calculus itself settles 'all the traditional concerns of logic' (p. 7). Accordingly, if we can find an axiomatization that is sound and complete with respect to Smiley consequence, then its rules will determine the meaning of the relevant constants - or as much of that meaning as is of proper concern to the logician. Smiley duly provides such an axiomatization, some of whose rules of course specify when a formula may be rejected, rather than specifying when it may be affirmed. His axiomatization includes, for instance, the rules 'From " $P$ infer $\sim P$ ' and 'From $\sim P$ infer " $P$ ' (p. 5). We can, then, see the meaning of a symbol like ' $~$ ' as being given, not only by its standard, affirmative, introduction and elimination rules, but by those rules in tandem with the appropriate rules for rejection.

\section{Calculi for which Smiley's solution is inadequate}

Smiley's way with the categoricity problem is highly attractive. As he says, however, 'the value of rejection as a solution to the general problem of sentential categoricity depends on one's being able to axiomatize at least the obvious candidates', and there are cases where his own method of axiomatization breaks down. That method rests upon ${ }^{6}$ a particular 'form of reductio ad absurdum (the dots stand for side premisses): if ...A $B$ and ... $A \vdash\left({ }^{*}\right) B$ then ... $\left(^{*}\right) A$ ' - where ' (*) $A$ ' signifies starring an unstarred formula or unstarring a starred one (p. 5). Accordingly, we can apply Smiley's method of axiomatization only to those calculi in which this form of reductio is sound, i.e. in which

$$
\text { if } X, A \vDash B \text { and } X, A \vDash(*) B \text {, then } X \vDash\left(^{*}\right) A \text {, }
$$

where $A$ and $B$ are arbitrary starred or unstarred formulae, where $X$ is an arbitrary set of such formulae, and where the comma indicates settheoretic union, so that $X, A$ is $X \cup\{A\}$.

Let us suppose, however, that we are dealing with a calculus that admits valuations in which some formulae are neither true nor false - i.e. that admits 'truth-value gaps' - and ask whether Smiley's version of reductio is sound for such a calculus. It ought to strike us that we have here two questions; for two notions of rejection, or of dissent from a proposition, can and must be distinguished in such circumstances. In identifying the first of these notions, it will help to quote the passage in which Smiley introduces

6 See especially p. 5, where this version of reductio is deemed 'the one principle that is always necessary for completeness, either as a primitive or a derived rule'. 
his own rejection sign:

A mechanism for rejection is there for anyone who wishes to use it, in the shape of an answer to a yes-or-no question. Questioner and answerer are usually different people, but if one puts the question to oneself, one comes up with the forms ' $P$ ? Yes' and ' $P$ ? No'. I suggest that '...? Yes' is a very passable realization of Frege's assertion-sign ..., and that '...? No' is an equally passable realization of a rejection-sign (p. 1).

Now it is reasonable to suppose that there will be an exact correspondence between declarative sentences which are to be evaluated as neither true nor false, and yes-or-no questions which are to be answered by neither 'yes' nor 'no'. Indeed, one might go so far as to claim that the plausibility of assessing 'The present King of France is bald' as neither true nor false rests upon the inappropriateness of giving either of the expected answers to the question 'Is the present King of France bald?'7 But, however that may be, a rejection sign which is explained in Smiley's way, as the correlate of the answer 'No', will precisely amount to an indication that the relevant proposition is being rejected as false. And this kind of rejection - internal rejection, as I shall call it - differs from the rejection of a proposition as merely being not true. If you do not believe that there is a present King of France, you will not wish to answer 'No' to the question 'Is the present King of France bald?'. You will, however, certainly refuse to give the answer 'Yes', and so in another perfectly good sense you will have dissented from, or rejected, the proposition expressed by 'The present King of France is bald'. Let us call this second, more inclusive, kind of rejection external rejection.

There is nothing paradoxical about there being two kinds of rejection in these cases. On the contrary. Smiley's strategy is to explain negation by invoking our prior understanding of the activity of rejecting (cf. p. 4). Since, when dealing with truth-value gaps, we certainly need to distinguish

7 If it could be defended, such a claim would provide a ground for assessing certain statements as neither true nor false that was independent of the desirability of giving 'a truth-functional account of the formation of complex statements by means of operators', and would thereby controvert a key thesis of Dummett 1959 (I quote from his p. 14). I shall not attempt any such defence here, but it is noteworthy that Dummett himself concedes 'that, if we had concerned ourselves with interrogative rather than assertoric force, we should have found a reason for distinguishing two undesignated truth-values for atomic sentences, even in accounting only for the use of these sentences on their own' (1981: 425). Moreover, his ground for not so concerning himself is none other than the claim that giving "the answer "No" [to a question] is precisely tantamount to, and is best analysed as, an assertion of the negation of the sentence uttered in interrogative form' (ibid., emphasis added). 
between internal and external negations ('The King of France isn't bald' vs 'It is not true that the King of France is bald'), it is unsurprising that we need to distinguish between internal and external modes of rejection. However, we need to ask which, if either, of these modes of rejection validates the version of reductio upon which Smiley bases his axiomatization, and here things are not as his discussion might lead one to expect. If '*' is understood as indicating external rejection, then the Smiley consequence relation must be understood as obtaining between $X$ and $A$ just in case every valuation that verifies all and only the formulae that appear unstarred in $X$ verifies the formula in $A$ (should it appear unstarred) or fails to verify it (should it appear starred). Accordingly, if we have that $X, A \vDash B$ and that $X, A \vDash(*) B$, we shall have that: every valuation that verifies all and only the formulae that appear unstarred in $X, A$ both verifies and fails to verify the formula in $B$. It follows that no valuation verifies all and only the formulae that appear unstarred in $X, A$, so that every valuation that verifies all and only the formulae that appear unstarred in $X$ will fail to verify the formula in $A$ (if it there appears unstarred) or will verify it (if it there appears starred). But the formula in $A$ there appears starred just in case it appears unstarred in (*)A, and vice versa, so that we have:

Every valuation that verifies all and only the formulae that appear unstarred in $X$ will verify the formula in $\left({ }^{*}\right) A$ (if it there appears unstarred), or will fail to verify it (if it there appears starred).

This is precisely the condition for $X \vDash(*) A$. Hence, Smiley's rule of reductio is sound when ' $*$ ' is given the external interpretation.

Matters stand otherwise, however, when it is given the internal interpretation. In this case, there is no call to revise the definition of Smiley consequence: we shall have $X \vDash A$ iff every admissible valuation that verifies all the formulae that appear unstarred in $X$, and falsifies all the formulae that appear starred, verifies the formula in $A$ (should it appear unstarred) or falsifies it (should it appear starred). Now from the premisses $X, A \vDash B$ and $X, A \vDash(*) B$, we can certainly infer that no valuation verifies all the formulae that appear unstarred in $X, A$ and falsifies all the formulae that there appear starred. However, we cannot conclude that every valuation that verifies all the formulae that appear unstarred in $X$, and falsifies all the formulae that there appear starred, verifies the formula in $\left(^{*}\right) A$ (should it appear unstarred) or falsifies it (should it appear starred). For it is possible that that formula is neither verified nor falsified. Indeed, with this in mind, a definite counter-example to the soundness of reductio is easy to construct. For consider a propositional calculus that conforms to the ordinary truth tables when all the sentence letters are assigned values, but which assigns no truth value to any formula containing a sentence 
letter to which no truth value has been assigned. In such a calculus we have that $P \& \sim P \vDash P$ and $P \& \sim P \vDash * P$, but we do not have $* *(P \& \sim P)$. (Any valuation that fails to assign a value to $P$ makes $(P \& \sim P)$ truth-valueless, rather than false.) Accordingly, internal rejection does not conform to Smiley's rule of reductio, and the logic of an internal negation sign (which corresponds to internal rejection) cannot be axiomatized in Smiley's way.

In fact, the situation is rather worse than this may suggest. We have been discussing the problem of axiomatizing the relationship of Smiley consequence in calculi that permit truth-value gaps, but that relationship was of interest only insofar as it determined sentential output, and in the calculi that now concern us, this determination no longer holds good. For consider (1) the classical, two-valued propositional calculus (whose Smiley consequence relation will be written as ' $k_{1}$ ') alongside (2) a supervaluational calculus that permits truth-value gaps but whose Smiley consequence relation $k_{2}$ is so defined that $X \vDash_{2} A$ just when $X \vDash_{1} A$. By definition, calculi (1) and (2) share a Smiley consequence relation, but they do not share a sentential output. In (1), there is no admissible valuation that neither verifies nor falsifies a sentence letter $P$; in (2) there is. And while (1) and (2) agree in respect of the traditional logical notions of contrariety, contradictoriness and the like (for the definitions of those notions in terms of Smiley consequence stand), that only brings out the need for some nontraditional notions to capture the differences between them. For example, in calculi (1) and (2), $P$ and $\sim P$ are contraries, for in neither calculus can both formulae be true. But in (2), unlike (1), both formulae can fail to be true.

\section{Categorical axiomatizations for simple calculi with truth-value gaps}

It lies far beyond the scope of this paper to try to solve the categoricity problem in general for calculi that permit truth-value gaps. I shall, however, present a straightforward solution for calculi of this kind that are simple - in the sense that they classify sentences simply as true, as false, or as neither - and that also exhibit a kind of compactness (to be spelled out in fn. 10). The argument of $\$ 2$ shows that before we can set about trying to find a suitable axiomatization, we need to know which semantical relationship we should be trying to axiomatize, and here a comparison with Smiley's procedure can help us. As he notes, for the sort of calculi that concern him 'the items [that specify a calculus's sentential output] are all of the form "It is possible/impossible to assign truth to every member of $\Gamma$ and falsehood to every member of $\Delta$ ", where $\Gamma$ and $\Delta$ exhaust the sentences' (p. 8). In other words, if we dispense with the modal operator in favour of quantification over valuations, each item appears in the form 
There is/isn't a valuation that assigns truth to every member of $\Gamma$ and falsehood to every member of $\Delta$.

In the calculi that concern us, it cannot be assumed that $\Gamma$ and $\Delta$ exhaust the well-formed formulae of the language. However, in the case of a simple calculus permitting truth-value gaps, the sentential output may be specified by a list of items in the form:

There is/isn't a valuation that assigns truth to every member of $\Gamma$, assigns falsehood to every member of $\Delta$, and assigns neither value to every member of $\mathrm{E}$,

where $\Gamma, \Delta$ and $\mathrm{E} d o$ exhaust the formulae. Now let us say that a valuation assigns an appropriate value to a starred or unstarred formula if the formula in question is valued true when unstarred or false when starred, and that it assigns an inappropriate value if the formula is valued false when unstarred or true when starred. Then we can define the Smiley multipleconclusion consequence relation $k_{\mathrm{m}}$ for a calculus to be the relation that obtains between two sets $X$ and $Y$ of starred or unstarred formulae just in case every valuation that assigns an appropriate value to every member of $X$ assigns an appropriate value to some member of $Y$. Accordingly, where $F$ is a set of formulae, where ${ }^{*} F$ is the result of prefacing every formula in $F$ with "*', and where the comma (on both sides of the consequence sign) indicates set-theoretical union, we shall have that $X F_{\mathrm{m}} F, " F$ just in case there is a valuation that assigns an appropriate value to every member of $X$ without assigning any value to any formula in $F$. Hence the statements

There is/isn't a valuation that assigns truth to every member of $\Gamma$, assigns falsehood to every member of $\Delta$, and assigns neither value to every member of $\mathrm{E}$,

are equivalent respectively to $\Gamma, " \Delta f_{\mathrm{m}} \mathrm{E}$, "E and to $\Gamma,{ }^{*} \Delta f_{\mathrm{m}} \mathrm{E}$, "E. So, if we can find axioms and rules which are sound and complete with respect to the $k_{\mathrm{m}}$ relation of a calculus, then we will have a categorical characterization of that calculus, for every item of the sentential output will be equivalent to a statement of deducibility or non-deducibility, so that the given axiomatization will exclude any other sentential output.

The reader may wonder whether the rejection sign is needed at all once the conclusion of the consequence relation is allowed to be a set of formulae rather than a formula, since this liberalization suffices to deal with the motivating example of $\$ 1$. In a classical multiple-conclusion calculus, we shall have $X \vDash \varnothing$ just in case no valuation verifies all the members of $X$, so that the rule 'From $A, \sim A$ infer $\varnothing$ ' excludes the valuation that verifies all the formulae of the language. Indeed, for a classical calculus, the use of a multiple-conclusion system constitutes an alternative solution to the cate- 
goricity problem. The principles $A, \sim A \vDash \varnothing$ and $\varnothing \vDash A, \sim A$ jointly dictate that $A$ and $\sim A$ are to be contradictories, and a classical calculus will admit a valuation in which all the formulae in $\Gamma$ are true and all the formula in $\Delta$ are false just when $\Gamma, \sim \Delta \not \varnothing .{ }^{8}$ Matters stand otherwise, however, with a system that permits truth-value gaps. A set of formulae $Y$ is a multipleconclusion consequence of a set of formulae $X$ if every valuation that verifies all the members of $X$ verifies at least one member of $Y$, but specifying the extent of this relation gives no information as to whether an unverified formula is false or is neither true nor false. Accordingly, the standard relation of multiple-conclusion consequence fails to determine the sentential output of a calculus that permits gaps. The sign of rejection is still needed.

It is clearly essential to the current proposal that the star should be understood as indicating internal rejection, and every subsequent occurrence of it in this paper is to be taken as such. For only when it is so taken do we get the required correspondence between starring a formula and evaluating it as false. We know, then, that an axiomatization of the $k_{\mathrm{m}}$ relation cannot be based upon a generalization of Smiley's rule of reductio, but reflection upon the circumstances in which that rule goes awry suggests the necessary emendation. As we saw, the inference from $X, A \vDash B$ and $X, A \vDash(*) B$ to $X \vDash\left({ }^{*}\right) A$ fails when '*' is understood internally because there might be a valuation that assigns an appropriate value to all the members of $X$ while assigning no value whatsoever to the formula in $A$. And this possibility can be excluded whenever every valuation that assigns some value to all the formulae in $X$ also assigns a value to the formula in $A$. Let us use the notation ' $[X]$ ' to stand for the set of formulae in $X$, and ' $[A]$ ' to stand for the formula in $A$; and let us say in such a case that $[X]$ determines $[A]$. Then, if we can find some appropriately syntactic way of specifying this relationship of determination, we shall be able to include as a sound rule this version of reductio:

From $X, A \vdash B$ and $X, A \vdash(*) B$ infer $\left.X \vdash^{*}\right) A$ whenever $[X]$ determines $[A]$.

These considerations generalize to the multiple-conclusion case. Let us say that one set of formulae determines another in a calculus just in case every admissible valuation of that calculus that assigns a truth value to all the members of the first set assigns a truth value to all the members of the second. Then, where $k_{\mathrm{m}}$ is the Smiley multiple-conclusion consequence

8 It is this insight that underpins the 'Do-It-Yourself' semantics of Hacking 1979, and explains why the treatment of classical logic given in that paper is not subject to the strictures of $\mathbb{1} 1$. For the rules that Hacking takes to determine the meanings of the constants are the rules of a sequent calculus, conceived as a metalogical theory of multiple-conclusion deducibility. 
relation for an arbitrary calculus, where $Z, Z^{\prime}, X, Y, Y^{\prime}$ are sets of starred or unstarred formulae, and where $A$ is a single starred or unstarred formula, we have that:

If $Z, X \vDash_{\mathrm{m}} A, Y$ and $Z^{\prime}, X \vDash_{\mathrm{m}}\left({ }^{*}\right) A, Y^{\prime}$, then $Z, Z^{\prime} \vDash_{\mathrm{m}}\left({ }^{*}\right) X, Y, Y^{\prime}$, so long as $\left[Z, Z^{\prime}\right]$ determines $[X]$.

For suppose not. Then there is a valuation $v$ that assigns appropriate values to all the members of $Z, Z^{\prime}$ while assigning no appropriate value to any member of $\left({ }^{*}\right) X, Y, Y^{\prime}$. Since $\left[Z, Z^{\prime}\right]$ determines $[X], v$ must assign inappropriate values to every member of $(*) X$, and hence must assign appropriate values to every member of $Z, X$ and to every member of $Z^{\prime}, X$. But since $Z, X \vDash_{\mathrm{m}} A, Y$ and $Z^{\prime}, X \vDash_{\mathrm{m}}\left({ }^{*}\right) A, Y^{\prime}$ - and since $v$ assigns no appropriate value to any member of $Y, Y^{\prime}-$ it must assign appropriate values to both $A$ and (")A. That, however, is impossible.

Where, then, ' $\vdash$ ' indicates multiple-conclusion deducibility, we are assured that the following rule will be sound with respect to any of the calculi that are under consideration:

Reductio If $Z, X \vdash A, Y$ and $Z^{\prime}, X \vdash\left(^{*}\right) A, Y^{\prime}$, then $\left.Z, Z^{\prime} \vdash{ }^{*}\right) X, Y, Y^{\prime}$, so long as $\left[Z, Z^{\prime}\right]$ determines $[X],{ }^{9}$

and, in tandem with the usual structural rules for multiple-conclusion systems, it is the cornerstone of our axiomatizations. Those structural rules are:

Overlap If $X$ overlaps $Y$, then $X \vdash Y$

Dilution If $X^{\prime} \vdash Y^{\prime}$, where $X^{\prime} \subset X$ and $Y^{\prime} \subset Y$, then $X \vdash Y$

Cut If - for some set $Z-X, Z_{1} \vdash Z_{2}, Y$ for every partition $\left\langle Z_{1}, Z_{2}\right\rangle$ of $Z$, then $X \vdash Y$,

(cf. Shoesmith and Smiley 1978, p. 29), and with Reductio they yield a useful rule of Dilemma. For suppose that $X,\left({ }^{*}\right) A \vdash Y$, where $A$ is a starred or unstarred formula. Then, by Dilution, we have $X,(*) A \vdash(*) A, Y$ and $\left.X,{ }^{*}\right) A \vdash A, Y$. By Reductio, this gives $X \vdash A, Y$ on condition that $[X]$ determines $[\{A\}]$. Accordingly, if $X, A \vdash Y$ and $X,(*) A \vdash Y$ then $X, A \vdash Y$ and $X \vdash A, Y$, so that by Cut we have $X \vdash Y$. $(\langle\varnothing,\{A\}\rangle$ and $\langle\{A\}, \varnothing\rangle$ are the only partitions of the set $\{A\})$. We have, in other words, the derived rule of

Dilemma If $X, A \vdash Y$ and $X,\left({ }^{*}\right) A \vdash Y$ then $X \vdash Y$, on condition that $[X]$ determines $[\{A\}]$.

Another consequence of Reductio is Ex Falso Quodlibet in the form: If

9 Here, and until further notice, I shall use 'determines' as a placeholder for the appropriate syntactic specification. Note that determination is a relation between sets of formulae of the relevant calculus, not between sets of starred or unstarred formulae. 
$X \vdash A$ and $\left.X \vdash{ }^{*}\right) A$ then $X \vdash Y$, for any set $Y$. (In Reductio, take $Z=Z^{\prime}=X$, $X=Y=Y^{\prime}=\varnothing, A=A$. Since, trivially, $[X]$ determines $\varnothing$, we have $\left.X \vdash{ }^{*}\right) \varnothing$, whence $X \vdash Y$, for any set $Y$, by Dilution.) It is possible to derive similarly a rule of reversal in the form:

Reversal If $X \vdash Y$ and $[Y]$ determines $[X]$ then $(*) Y \vdash(*) X$.

These rules suffice to establish the main lemma in a Henkin-style completeness proof - namely, that any consistent set of starred or unstarred formulae may be extended to produce another consistent set that is in a suitable way maximal. More exactly, if we suppose that the deducibility relation is compact in the sense that whenever $U \vdash V$ then $U^{\prime} \vdash V$ for some finite subset $U^{\prime}$ of $U$, we have the following

Lemma: For any sets of starred or unstarred formulae $X$ and $Y$ such that $X \nvdash Y$, there is a set $\bar{X} \supset X$ for which $\bar{X} \nvdash Y$, and such that for any formula $\Phi$ determined by $[X]$, either the corresponding unstarred formula $\Phi$ is a member of $\bar{X}$ or the corresponding starred formula ${ }^{*} \Phi$ is. Moreover, $\bar{X}$ is closed with respect to formulae that $[X]$ determines, in the sense that for any such formula, a corresponding starred or unstarred formula is deducible from $\bar{X}$ only if it is a member of $\bar{X}$.

Proof: Enumerafe the formulae of the language as $\Phi_{1}, \Phi_{2}, \ldots$, and define a sequence of sets $\left\{X_{1}, X_{2}, \ldots\right.$ of starred or unstarred formulae according to
the rule:

$$
\begin{array}{lll}
X_{0}=X & & \\
& X_{n}, \Phi_{n} & \text { if } X_{n}, \Phi_{n} \nvdash Y \\
X_{n+1}= & X_{n},{ }^{*} \Phi_{n} & \text { if } X_{\mathrm{n}},{ }^{*} \Phi_{n} \nvdash Y \\
X_{n}, & \text { otherwise }
\end{array}
$$

Then define $\bar{X}$ to be the union of all the $X_{n}$. It is plain from the construction that $X_{n} \nLeftarrow Y$ for every $n$, so that $\bar{X} \nvdash Y$ by the compactness of the deducibility relation. To establish that $\bar{X}$ possesses the relevant kind of maximality, suppose that neither $\Phi$ nor " $\Phi$ is in $\bar{X}$, where $\Phi$ is, for definiteness, $\Phi_{n}$. That can only be because $X_{n+1}=X_{n}$, whence $X_{n}, \Phi \vdash Y$ and $X_{n},{ }^{*} \Phi \vdash Y$. Now if $[X]$ determines $\left\{\Phi_{n}\right\}$, so does $\left[X_{n}\right]$, whence $X_{n} \vdash Y$, by Dilemma, contrary to $X_{n} \nvdash Y$. Thus $[X]$ cannot determine $\left\{\Phi_{n}\right\}$, alias $\{\Phi\}$. That is to say, for any $\Phi$ for which $[X]$ determines $\{\Phi\}$, either $\Phi \in \bar{X}$ or " $\Phi \in \bar{X}$. To show that $\bar{X}$ is closed in the relevant sense, suppose that $[X]$ determines $[\{A\}]$, that $\bar{X} \vdash A$, but that $A \notin \bar{X}$. By maximality, $(*) A \in \bar{X}$, whence $\left.\bar{X} \vdash{ }^{*}\right) A$ and $\bar{X} \vdash Y$, by Ex Falso Quodlibet, contradicting $X \nvdash Y$. Q.E.D.

In selecting a calculus with which to illustrate the application of this lemma in proving completeness, I can do no better than to choose a propositional calculus that is a fragment of a predicate system presented in 
ANALYSIS for 1960 by Smiley himself - although it is worth stressing that the method of proof extends straightforwardly to the predicate case, and hence yields a categorical axiomatization for Smiley's version of the free first-order calculus. ${ }^{10}$ The primitive symbols of this fragment are: a sign for (internal) negation ' $\sim$ ' - defined so that $\sim A$ is false when $A$ is true, and is true when $A$ is false, and is otherwise left without a truth value; a sign for (internal) conjunction ' $\&$ ' - defined so that $A \& B$ is true when $A$ and $B$ are both true, is false when one of $A$ and $B$ is false and the other is either true or false, and is otherwise left without a value; and a unary operator $T$, to be read 'it is true that' - defined so that TA is true if $A$ is true, and is false in every other case. As Smiley remarks (1960: 131), this system makes possible the rigorous definition of the notion of presupposition that concerned Strawson and his followers; and the external connectives are definable within it using the relevant internal connective together with the truth operator. Thus the external negation of $A$ may be identified with $\sim T A$.

In formulating rules which comprise a sound and complete axiomatization of the Smiley multiple-conclusion consequence relation for this calculus, and hence constitute a categorical axiomatization of it, the first task is to specify the determination relation in an appropriately syntactic way. Let us say that one formula occurs vulnerably in another if the first occurs in the second, but outside the scope of the $T$ operator. Then it is clear from the semantical rules of the calculus that $[X]$ determines $[Y]$ just in case every sentence letter that occurs vulnerably in some formula in $[Y]$ also occurs vulnerably in some formula in $[X]$. This specification should be understood to replace the term 'determines' in the formulation of Reductio, Dilemma and Reversal; for, as I said, that term was no more than a placeholder. And we get a complete axiomatization of our calculus by appending to those rules, so understood, the structural rules together with the following rules for the individual connectives:
1. From $\sim A$ infer $* A$
2. From $* A$ infer $\sim A$
3. From $A \& B$ infer $A$

10 The method also applies to a number of other simple calculi admitting truth-value gaps, but it is limited by the requirement that the deducibility relation shall be compact. Such a relation can be sound and complete only for calculi which display the semantical analogue of this kind of compactness, as some calculi admitting truthvalue gaps do not. (If, for example, the language of a calculus contains an infinite number of sentence letters, and no valuation is permitted to assign truth to every sentence letter, but everything else is standard, then the empty set is a semantical consequence of the set of all sentence letters, but not of any finite subset of it.) All the same, this limitation does not prevent the method from working across a broad and central class of cases. 
4. From $A \& B$ infer $B$

5. From $A, B$ infer $A \& B$

6. From $A$ infer $T A$

7. From $T A$ infer $A$.

It should be noted that $(5)$ yields 'From * $(A \& B)$ infer * $A,{ }^{*} B$ ' by Reversal (because $\{A \& B\}$ determines $\{A, B\}$ ), but that (3) does not yield 'From $* A$ infer* $(A \& B)$ ' $(\{A\}$ does not determine $\{A \& B\})$. Similarly, while (7) yields 'From " $A$ infer " $T A$ ' (because $\{A\}$ determines $\{T A\}),(6)$ does not yield 'From *TA infer *A' $(\{T A\}$ does not determine $\{A\})$. However, $\{A\}$ determines $\{\sim A\}$ and vice versa, so we can apply Reversal to establish the inter-deducibility of a formula and its double negation. For, reversing (1), we have 'From $A$ infer * $\sim A$ ' and, from (2), 'From * $\sim A$ infer $\sim \sim A$ '; while from (1), we have 'From $\sim \sim A$ infer * $\sim A$ and, from reversing (2), 'From * $\sim A$ infer $A$ '. It should also be noted that the deducibility relation corresponding to these rules is compact in the sense specified above, so that the lemma is applicable.

In establishing the completeness of these rules, we suppose that $X \Vdash Y$, and note that the lemma asserts the existence of a set $\bar{X}$ which includes $X$, for which $\bar{X} \nvdash Y$, and which is closed with respect to formulae that $[X]$ determines. We then define a valuation $v$ such that, for any sentence letter $P$,

$v(P)=\mathrm{T}$ iff $[X]$ determines $\{P\}$ and $P \in \bar{X}$

$v(P)=\mathrm{F}$ iff $[X]$ determines $\{P\}$ and ${ }^{*} P \in \bar{X}$.

That is to say, any sentence letter undetermined by $[X], v$ leaves without an assignment. We then have the following

Theorem: (a) For any formula $A$ such that $[X]$ determines $\{A\}, v(A)=\mathrm{T}$ iff $A \in \bar{X}, v(A)=\mathrm{F}$ iff $* A \in \bar{X}$;

(b) for any formula $A$ such that $[X]$ does not determine $\{A\}, v(A) \neq \mathrm{T}$ and $v(A) \neq \mathrm{F}$.

Completeness follows directly from this theorem. The valuation $v$ plainly assigns appropriate values to all the members of $X$. However, it does not assign an appropriate value to any member of $Y$. For if an arbitrary formula $B$ in $[Y]$ is not determined by $[X]$, then $v$ assigns it no value at all. While if $B$ is determined by $[X]$, and (for example) appears unstarred in $Y$, then if $v$ gave it an appropriate value, it would value it as $\mathrm{T}$, showing by the theorem that $B \in \bar{X}$, whence $\bar{X} \vdash Y$ by Overlap, contradicting the assumption that $X \nvdash Y$. (The argument is similar if $B$ appears starred.) That is to say, if $X \nvdash Y$, then $X F_{\mathrm{m}} Y$, which is precisely the condition for completeness with respect to multiple-conclusion consequence.

The proof of the theorem is, as usual, by induction on the complexity of the formula $A$. The base case follows directly from the definition of $v$, and we have three inductive cases: 
Case 1: $A=\sim B$. For $(\mathrm{a})$ we note that $[X]$ determines $\{A\}$ iff $[X]$ determines $\{B\}$. So for determined $A$ we can argue: $(1) v(A)=\mathrm{T}$ iff $v(B)=\mathrm{F}$ iff $* B \in \bar{X}$ (by the inductive hypothesis) iff $\sim B \in \bar{X}$ (by rules 1 and 2) iff $A \in \bar{X}$; (2) $v(A)=\mathrm{F}$ iff $v(B)=\mathrm{T}$ iff $B \in \bar{X}$ (by ind. hyp.) iff $\sim \sim B \in \bar{X}$ (see above) iff $\sim A \in \bar{X}$ iff $* A \in \bar{X}$ (by rules 1 and 2 ).

For (b), suppose e.g. that $v(A)=\mathrm{T}$ but that $[X]$ does not determine $\{A\}$. Then $v(B)=\mathrm{F}$ even though $[X]$ does not determine $\{B\}$, contradicting the inductive hypothesis.

Case 2: $A=B \& C$. (a) $([X]$ determines $\{A\}$ and $v(A)=\mathrm{T})$ iff $([X]$ determines $\{B\}$ and $[X]$ determines $\{C\}$ and $v(B)=v(C)=\mathrm{T})$ iff $(B \in \bar{X}$ and $C \in \bar{X}$ ) (by inductive hypothesis) iff $A \in \bar{X}$ (by rules $3,4,5$ ). Also ([X] determines $\{A\}$ and $v(A)=\mathrm{F})$ iff $([X]$ determines $\{B\}$ and $[X]$ determines $\{C\}$ and either $v(B)=\mathrm{F}$ while $v(C)=\mathrm{T} / \mathrm{F}$ or $v(C)=\mathrm{F}$ while $v(B)=\mathrm{T} / \mathrm{F})$ iff $(* B \in \bar{X}$ and either $C \in \bar{X}$ or ${ }^{*} C \in \bar{X}$; or ${ }^{*} C \in \bar{X}$ and either $B \in \bar{X}$ or ${ }^{*} B \in \bar{X}$ ). Now by Dilution of rules 3 and 4 we have 'From $A \& B$ infer $A, B$ ', 'From $A \& B$ infer $A, * B$ ', 'From $A \& B$ infer $* A, B$ ' whence by Reversal 'From * $A, * B$ infer * $(A \& B)$ ', 'From * $A, B$ infer * $(A \& B)$ ', and 'From $A,{ }^{*} B$ infer $*(A \& B)$ '. Hence the chain of biconditionals can be extended thus: iff $*(B \& C) \in \bar{X}$ iff $* A \in \bar{X}$, as required.

For (b), suppose that $v(A)=\mathrm{T} / \mathrm{F}$ but that $[X]$ does not determine $\{A\}$. Then $v(B)=\mathrm{T} / \mathrm{F}$ and $v(C)=\mathrm{T} / \mathrm{F}$ even though $[X]$ fails to determine either $\{B\}$ or $\{C\}$, contradicting the inductive hypothesis.

Case 3: $A=T B$. Then $[X]$ determines $\{A\}$ irrespective of the status of $B$, so we have only to establish clause (a). Now $v(T B)=\mathrm{T}$ iff $v(B)=\mathrm{T}$ iff $B \in \bar{X}$ (by inductive hypothesis) iff $T B \in \bar{X}$ (by rules 6 and 7). And $v(T B)=F$ iff $* T B \in \bar{X}$ follows directly from maximality.

This completes the proof of the theorem, and with it the identification of rules that are categorical for the calculus under consideration. ${ }^{11}$

The University of Michigan Ann Arbor, MI, 48109-1003, USA rumfitt@umich.edu

\section{References}

Dummett, M. 1959. Truth. Reprinted in his Truth and Other Enigmas. London: Duckworth, 1978, 1-19.

Dummett, M. 1981. Frege: Philosophy of Language, second edition. London: Duckworth.

Hacking. I. 1979. What is Logic? Journal of Philosophy 76: 285-319.

11 I am grateful to Professors Timothy Smiley and Timothy Williamson for helpful comments on a draft. 


\section{IAN RUMFITT}

Shoesmith, D. J. and T. J. Smiley. 1978. Multiple-conclusion Logic. Cambridge: Cambridge University Press.

Smiley, T. J. 1960. Sense without denotation. Analysis 20: 125-35.

Smiley, T. J. 1996. Rejection. Analysis 56: 1-9. 